\title{
AN INVERSE DYNAMICS MODEL FOR THE ANALYSIS, RECONSTRUCTION AND PREDICTION OF BIPEDAL WALKING
}

\author{
Bart Koopman, Henk J. Grootenboer and Henk J. de Jongh \\ University of Twente, Faculty of Mechanical Engineering, Laboratory of Biomedical Engineering, \\ P.O. Box 217, 7500 AE Enschede, The Netherlands
}

\begin{abstract}
Walking is a constrained movement which may best be observed during the double stance phase when both feet contact the floor. When analyzing a measured movement with an inverse dynamics model, a violation of these constraints will always occur due to measuring errors and deviations of the segments model from reality, leading to inconsistent results. Consistency is obtained by implementing the constraints into the model. This makes it possible to combine the inverse dynamics model with optimization techniques in order to predict walking patterns or to reconstruct non-measured rotations when only a part of the three-dimensional joint rotations is measured. In this paper the outlines of the extended inverse dynamics method are presented, the constraints which define walking are defined and the optimization procedure is described. The model is applied to analyze a normal walking pattern of which only the hip, knee and ankle flexions/extensions are measured. This input movement is reconstructed to a kinematically and dynamically consistent three-dimensional movement, and the joint forces (including the ground reaction forces) and joint moments of force, needed to bring about this movement are estimated.
\end{abstract}

\section{INTRODUCTION}

Numerous models have been developed to simulate human walking, based on segment models in varying complexity from three (McMahon, 1984) up to 17 segments (Hatze, 1981a). Symmetry between the right and left leg is often assumed to reduce complexity (e.g. Brand et al., 1982), and the movement is often restricted to the sagittal plane only.

The two ways to apply the equations of motion to the segments model are usually referred to as the 'direct dynamics method' and the 'inverse dynamics method'. In the direct dynamics method, the movements of the segments are calculated by integrating the equations of motion. This is only possible when the joint moments of force are known or assumed to be zero. The latter is the case in ballistic walking (McMahon, 1984). It is also possible to choose for the internal forces such values that a normal walking pattern results (e.g. Pandy and Berme, 1988) or to calculate the joint moments of force from estimations of the muscle forces (e.g. Olney and Winter, 1985).

In the inverse dynamics method, the joint forces and joint moments of force are calculated from a prescribed movement. Since the segmental movements, in contrast to the internal forces, can be measured, this method is commonly applied for the analysis of measured movements. Hereby the measured ground reaction forces are used as an input for most of these models (e.g. Brand et al., 1982). This, however, is not a necessity for a bipedal model, as was shown by Hardt and Mann (1980). The ground reaction forces

Received in final form 21 March 1994. are, except for the double support phase, completely determined by the segmental displacements. However, since the measured displacements have to be differentiated twice with respect to time in order to obtain the accelerations, this may result in large numerical errors when no precautions are taken. To avoid these errors, Chao and Rim (1973) combined optimization techniques with the direct dynamics method to calculate the joint moments of force in normal walking.

Optimization techniques are also used in predictive models, where both the movement and the internal forces have to be calculated. This approach may find a wide application in rehabilitation technology, for example, to calculate the effect of prosthetic components on the walking pattern or in functional electrical stimulation. Chow and Jacobson (1971) were the first to develop a (semi) predictive model for walking: with prescribed hip trajectories, ground reaction forces and ankle moments of force they predicted the hip and knee angles and moments of force by minimizing a criterion reflecting the mechanical energy expenditure. The complex model of Hatze (1981b) includes muscle dynamics and is applied to predict the long jump.

The predictive model presented here is, in contrast to other existing models, based on a combination of inverse dynamics and optimization techniques. There are two reasons for choosing the inverse dynamics method instead of the direct dynamics method: first, since movements can be measured and internal forces and moments of force cannot, the inverse dynamics part of the model can be used separately for the gait analysis of measurement. Second, walking is a constrained movement, and it is easier to implement kinematic constraints in an inverse method than in 
a direct method. One kinematic constraint is, for example, that the feet must be exactly on the floor during the stance phase. However, the distinction between inverse and direct dynamics is not essential since both methods should eventually yield the same results.

Dynamic constraints are applied to achieve that the segments model is in balance for the complete walking cycle, which may best be explained in the frontal plane (Fig. 1). With an input consisting of the sagittal hip, knee and ankle rotations only, the foot will move straight under the hip joint. Since the ground reaction forces can only apply to the foot and are in general not in the line of action of the static and dynamic forces acting on the total center of mass of the segments model, an imbalance moment $M$ is necessary to keep the body upright [Fig. 1(a)]. This imbalance is reduced by estimating the leg adduction in an optimization scheme and thus meeting the dynamic constraints [Fig. 1(b)].

The application of kinematic and dynamic constraints eventually results in a movement of the segments model which is consistent with physical demands: all deviations due to measuring errors and approximations in the segments model are corrected for. As an example, the model is applied to a normal gait pattern which is basically two-dimensional: only the hip, knee and ankle flexions/extensions are measured. The non-measured rotations are estimated with the constraints, and the resulting three-dimensional movement is analyzed.

\section{METHODS}

\section{The segments model}

In the segments model the human body is modeled as a coupled system of right bodies containing eight
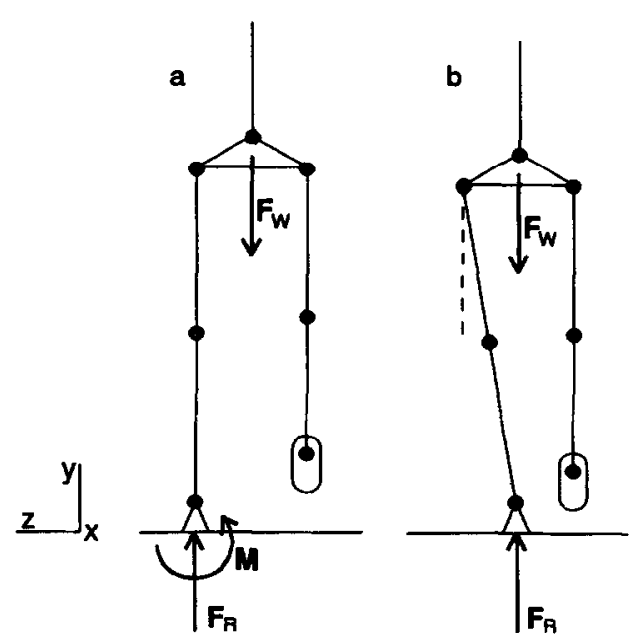

Fig. 1. Imbalance of the segments model. The imbalance moment $M$ can be viewed as resulting from an eccentricity of the ground reaction force $F_{R}$ with the body weight $F_{w}$ (a) and is corrected with a hip adduction (b). segments (Fig. 2): the upper legs, lower legs, feet, pelvis and the head, arms and trunk (HAT) segment. The number of segments is a compromise between the wish to avoid unnecessary complexity and large computation times, on the one hand, and to simulate the movement adequately on the other hand. The segments are connected in the joints; the point of contact between the foot and the floor is modeled as if it were a joint as well. In this view, the floor is a segment with zero velocity and infinite mass, which makes it possible to calculate the ground reaction forces in the same way as the joint forces. A reference frame is attached to the floor, with the $x$-axis pointing in the walking direction, the $y$-axis pointing upward and the $z$-axis perpendicular to the $x y$-plane in the lateral direction (Fig. 2).

In each segment a local frame is defined according to the method used by Brand et al. (1982), which is based on the location of some anatomical landmarks. It is extended with definitions for the segments of the feet and the HAT segment. A detailed description of the construction of these frames, as well as a list of all segmental parameters, has been given elsewhere (Koopman, 1989).

The mass, the position of the center of mass in the local frame and the moment of inertia tensor are determined with the regression equations of Chandler et al. (1975). These relations depend on the local dimensions of the segments and the total body weight, and are in some respects adapted to the segments model used in this study: the properties for the footsegment are corrected for the influence of the shoe, and a distinction is made for the HAT and the pelvis.
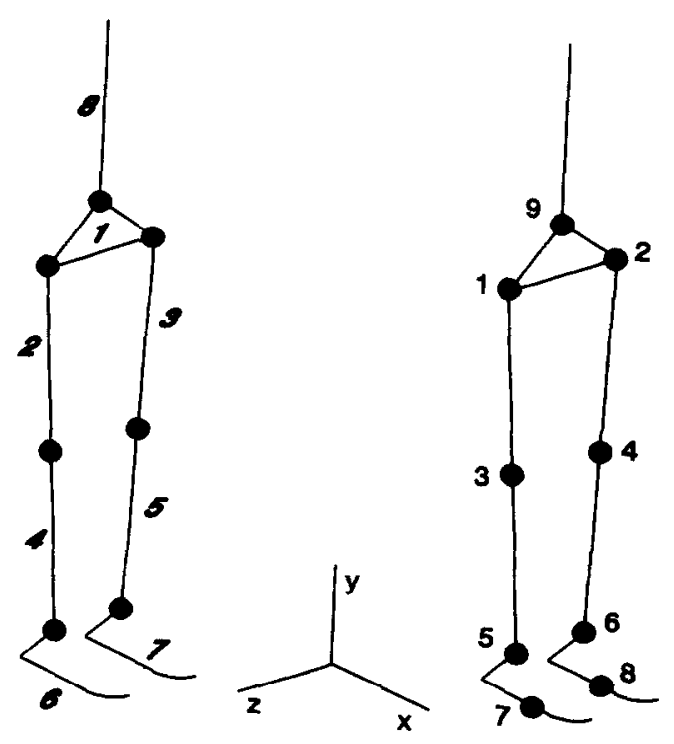

Fig. 2. The segments model for the body at rest, with segments: pelvis (1), R-L femur (2-3), R-L tibia (4-5), R-L foot (6-7) and HAT (8); with joints: R-L hip (1-2), R-L knee (3-4), R-L ankle (5-6), R-L foot-floor (7-8) and HAT-pelvis (9); and the definition of the reference frame: forward axis $(x)$, vertical axis $(y)$ and lateral axis $(z)$. 
It is assumed that the axes of the local frames of the segments are in the principal directions, so that the principal moments of inertia as determined by Chandler et al. can be used.

All joints are modeled as potential ball-hinges with three independent rotational degrees of freedom. However, the nature of the joint (ball-hinge, linehinge) is determined by the input joint rotations of the inverse dynamics model and the constraints: when the joint angular velocity vector has a fixed direction, the joint will effectively act as a line-hinge. The positions of the joints in the local frames of the segments may depend on the joint rotation and thus vary implicitly with time, so joint translations are allowed but only as functions of the joint rotations.

An example of this is the modeling of the roll-over of the foot segment: the point of contact between foot and floor shifts from heel to toe during stance. It is assumed that the instantaneous point of contact can be defined as the point where the resultant ground reaction forces apply to the foot and that the location of this point in the local frame of the foot depends on the rotation of the foot relative to the floor only. The set of all contact points defines the effective shape of the (deformed) foot. From a defined foot shape it is possible to calculate the location of the contact point in foot coordinates as well as in floor coordinates as a function of the foot rotation. The foot shape that is used in this study is based on measurements and is shown in Fig. 3. Not allowing for independent joint translations effectively reduces the number of independent degrees of freedom.

\section{Construction of the movement}

The movement of a segment is the combination of translation and orientation of the local frame relative to the reference frame. The orientation is uniquely defined by a rotation tensor, its components are calculated using Euler parameters to assure orthogonality (Koopman, 1989). Rotations are presented in clinical angles.
With known joint rotations, all segmental movements are defined relative to an arbitrary reference point and with an unknown reference orientation. These are constructed by applying the kinematic constraints.

- The reference orientation (as a function of time) is deduced from the demands that at the time of heel contact, both feet touch the floor and during a part of the single stance phase (foot flat) the rotation of the foot relative to the floor is zero.

- The displacement of the reference point is calculated from the constraint that during the stance phase no slipping between foot and floor may occur. Other kinematic constraints check for the kinematic consistency of the movement and, if necessary, correct for these:

-During the double support phase both feet must remain on the floor and no slipping between foot and floor may occur. Due to measuring errors, deviations of the segments model or in this case simply the fact that not all joint rotations are measured, this constraint will always be violated. The necessary corrections are preferably performed on rotations which are not measured, in this case on the pelvic segmental rotations around the forward and vertical axes.

-During the swing phase the foot must remain above the floor and the legs may not collide. Also here-to corrections are performed on the not-measured rotations (i.e. pelvic rotations).

- The calculated velocity is corrected for the measured velocity by assuming a sinusoidal pelvic rotation around the vertical axis.

\section{Kinetics}

The ground reaction forces are unequivocally determined by the movements of the segments, e.g. the acceleration of the total center of mass, with one exception: the force component that acts on the straight line between the two contact points of the feet with the floor. It is assumed that this counteracting force is zero. Instead of defining four sets of force



Fig. 3. Lateral view of the foot shape in local foot coordinates with the ankle joint at the origin $x=y=0$. 
equations for each phase of the walking cycle (e.g. Hardt and Mann, 1980), a shift function $f$ is introduced which defines the shift of body weight from one foot to the other during the double support phase; $f$ equals one during single support and zero during the swing phase. The set of vector equations to solve the joint forces and ground reaction forces becomes

$$
\begin{aligned}
\sum_{j=1}^{N_{i j}} \mathbf{F}_{i j} & =m_{i}\left(\ddot{\mathbf{x}}_{i}-\mathbf{g}\right) \\
\mathbf{F}_{\mathrm{R}} & =f \sum_{i=1}^{N_{\mathbf{s}}} m_{i}\left(\ddot{\mathbf{x}}_{i}-\mathbf{g}\right), \\
\mathbf{F}_{\mathbf{L}} & =(1-f) \sum_{i=1}^{N_{\mathbf{s}}} m_{i}\left(\ddot{\mathbf{x}}_{i}-\mathbf{g}\right),
\end{aligned}
$$

where $\mathbf{F}_{i j}$ is the force vector in joint $j$ acting on segment $i, m_{i}$ is the mass, $\ddot{x}_{i}$ is the acceleration vector of the center of mass, $g$ is the acceleration of gravity vector, $N_{i j}$ is the number of joints at segment $i, N_{\mathrm{s}}$ is the number of segments and $F_{R}$ and $F_{L}$ are the right and left floor reaction force vectors. The equations for the left and right floor reaction forces are equivalent. Likewise, the equations for the joint moments of force are

$$
\begin{array}{r}
\sum_{j=1}^{N_{i j}}\left(\mathbf{M}_{i j}+\mathbf{r}_{i j} \times \mathbf{F}_{i j}\right)=\frac{\mathrm{d}\left(\mathbf{J}_{i} \cdot \boldsymbol{\omega}_{i}\right)}{\mathrm{d} t}, \\
\mathbf{M}_{\mathbf{R}}=f \mathbf{M}_{\mathbf{G}} \\
=f\left(\sum_{i=1}^{N_{\mathbf{L}}} \frac{\mathrm{d}\left(\mathbf{J}_{i} \cdot \boldsymbol{\omega}_{i}\right)}{\mathrm{d} t}-\mathbf{r}_{\mathrm{R}} \times \mathbf{F}_{\mathrm{R}}-\mathbf{r}_{\mathbf{L}} \times \mathbf{F}_{\mathbf{L}}\right),
\end{array}
$$

where $\mathbf{M}_{i j}$ is the moment of force vector in joint $j$ working on segment $i, J_{i}$ is the inertia tensor, $\omega_{i}$ is the rotation velocity vector, and $\mathbf{r}_{i j}$ is the vector pointing from the center of mass to joint $j . \mathbf{M}_{\mathrm{G}}$ is the total ground reaction moment of force vector which must be zero except for the vertical component that represents the friction between feet and floor. The horizontal components of $\mathbf{M}_{\mathbf{G}}$ represent the imbalance of the segments model that is dealt with in the dynamic constraints.

\section{Optimization}

In general, the optimization problem can be stated as follows. Find, in subsequent iterations, estimates for the rotations that are not measured by minimizing an objective function. The measured joint rotations have fixed values, while the non-measured rotations are initially zero. The solution should not violate any constraints.

The joint and segmental rotations are represented by finite Fourier series. One of the reasons for this representation is that the Fourier coefficients provide a relatively small set of optimization variables, which are necessary to avoid large computation times. Furthermore, the movement is implicitly cyclic and con- tinuous and at each point of time of the exact history and future of the movement this is known.

The number of Fourier coefficients is chosen such that the difference between the Fourier series representation and the actual, measured and averaged rotation is very small compared to the measuring and averaging deviations. In practice, a representation up to the 10 th waveform, resulting in a total of 21 Fourier coefficients, is for most walking movements sufficient.

Instead of using a minimal energy criterion for the objective function, such as proposed by Chow and Jacobson (1971) and also used by Vaughan et al. $(1982 \mathrm{a}, \mathrm{b})$, a slightly modified criterion, $U$, is minimized:

$$
U=\cdot \sum_{j=1}^{N_{j}} \sum_{k=1}^{3} \frac{1}{T} \int_{\tau=0}^{T} \alpha_{j k}\left(\frac{\mathbf{M}_{j k}}{\mathbf{M}_{\max , j k}}\right) \beta \mathrm{d} \tau,
$$

where $k$ denotes the coordinate number, $T$ is the stride time, $\mathbf{M}_{j \mathbf{k}}$ is the calculated joint moment of force and $\mathbf{M}_{\max , j k}$ is (depending on the sign of $\mathbf{M}_{j k}$ ) the maximal positive or negative isometric joint moment of force, obtained from the literature (Koopman, 1989) or based on the results of muscle models (Thunnissen, 1993). $\alpha_{j k}$ and $\beta$ are optimization parameters, with $\alpha_{j k}$ equal to one or zero (when no joint rotation in direction $k$ is allowed) and $\beta$ has a chosen value of 2 . This criterion shows a large resemblance with the fatigue criteria used by Crowninshield and Brand (1981) and Dul et al. (1984a, b) to determine the load sharing between synergistic muscles. In criterion (3) the load sharing between the joints is based on the properties of the joints. Therefore, this criterion will also be referred to as a fatigue criterion.

Kinematic constraints are used to construct a consistent movement for each iteration of the optimization problem. Other kinematic constraints are implemented for the optimization only and define the range in which the solution has to be found.

- The ranges of motion in each joint define allowable joint angles.

-Allowable ranges for the Fourier coefficients of the rotations restrict the maximal angular accelerations.

A violation of these constraints results in an increase of a penalty function that is multiplied with the objective function $U$.

With the dynamic constraints, the imbalance moment of force $\mathbf{M}_{\mathbf{G}}$ is minimized. This is implemented as a dimensionless time integral of the components of $\mathbf{M}_{G}$, weighted with moments of force which represent an allowable eccentricity between the calculated and prescribed contact points on the surface of the foot. This time integral is added to the objective function as a penalty function. As a result, the solution of the optimization is both kinematically and dynamically consistent.

The optimization is performed with standard numerical routines utilizing a quasi-Newton algorithm (Numerical Algorithms Group, 1984), which is modified for this specific problem. 
Measured, constructed, reconstructed and predicted rotations

For each leg three electrical goniometers are used to measure the hip, knee and ankle flexions/extensions as functions of time. The goniometers are connected to each other in a light-weight external frame which is attached to the leg, with the axes of rotation positioned in the assumed joint axes of rotation. To allow for variations in the directions and positions of the joint axes of rotation, the external frame is not rigid but consists of rods and tubes that can slide relative to each other.

The time parameters of the walking movement, which are determined with the points of time of heel contact (HC) and toe-off (TO), are measured with electrical foot contact switches. Under each foot, two switches are placed in parallel, one at the heel and one joint in front of the ball of the foot.

The output voltages are measured on a personal computer with a sampling rate of $200 \mathrm{~Hz}$. The subject of the experiment is asked to walk a distance of about $25 \mathrm{~m}$ at a comfortable speed; the measurements take place during $10 \mathrm{~s}$ in the middle part of the walkway. Here the average velocity is also measured.

A normal walking pattern of a healthy subject (male, $24 \mathrm{yr}, 83 \mathrm{~kg}, 1.83 \mathrm{~m}$ ) is measured as an example to be analyzed. Of this subject, some anatomical dimensions were measured to define the segments model. The data of subsequent cycles are averaged following standard procedures (Koopman, 1989), as well as the data for left and right leg, for which symmetry is assumed. The average joint rotations with their standard deviations are shown in Fig. 4.

From the non-measured rotations, the pelvic rotations are constructed with the kinematic constraints. The hip adduction and the HAT rotation around the lateral axis are reconstructed mostly from the dynamic constraints. Minimization of the fatigue criterion $U$ predicts the HAT rotations around the vertical and forward axes. The other rotations (knee and ankle ad/abduction, leg joints endo/exorotations) are not optimized and keep their initial zero values.

\section{RESULTS}

The components of the imbalance moment $\mathbf{M}_{\mathbf{G}}$ in the forward $(x$-)direction and lateral (z-)direction reach maximal values of about 120 and $80 \mathrm{Nm}$ respectively, which cannot be neglected in comparison to the joint moments of force (i.e. the ankle plantar flexion moment has a maximum at about $120 \mathrm{Nm}$ ). Relatively small joint rotations are sufficient to reduce the imbalance and fulfil the dynamic constraints, as is shown in Fig. 5. The reconstructed hip adduction is such that the foot is placed straight under the middle of the pelvis during the stance phase. During the swing phase, the leg tends to swing straight under the hip joint [Fig. 5(a)]. The reconstructed HAT rotation shows that the segments model has to lean forward in order to keep balance [(Fig. 5(b)]. The dynamic component of the forward rotation, represented by the amplitude, is essential in the balancing process. Small angular fluctuations result in large angular accelerations due to the relatively large moment of inertia of the HAT segment. The reconstructed rotations agree well with measurements, reported in the literature (e.g. Inman et al., 1981; Kadaba et al., 1990), at least where the tendencies are concerned. The rotations' offsets may differ more which is mainly caused by the different definitions of the offsets in the local experimental setups.

The pelvic rotations around the forward ( $x$-)axis and vertical ( $y$ )axis [Figs. 6(a) and (b)] are determined by the kinematic constraints: the $x$-rotation is necessary to keep both feet on the floor during the double support phase and the $y$-rotation to increase the step length and meet the prescribed average velocity. There is a good agreement with measured rotations, found in the literature (e.g. Inman et al., 1981; Kadaba et al., 1990; Vink and Karssemeijer, 1988): the general


Fig. 4. Measured average joint rotations: hip flexion (a), knee extension (b) and ankle dorsiflexion (c) with standard deviations during one cycle. The support phase is from $0-60 \%$, the swing phase from 60 to $100 \%$, and the double support phases from 0 to $10 \%$ and from 50 to $60 \%$. 

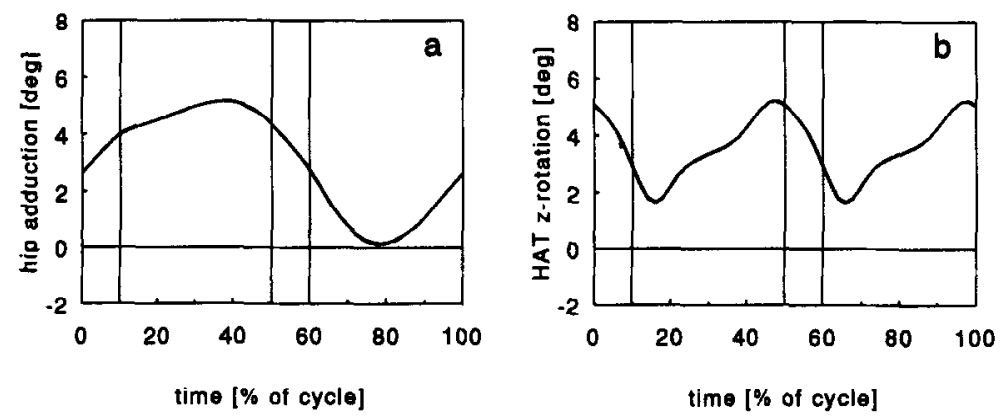

Fig. 5. Hip adduction (a) and HAT segment forward (z-) rotation (b) reconstructed with dynamic constraints.
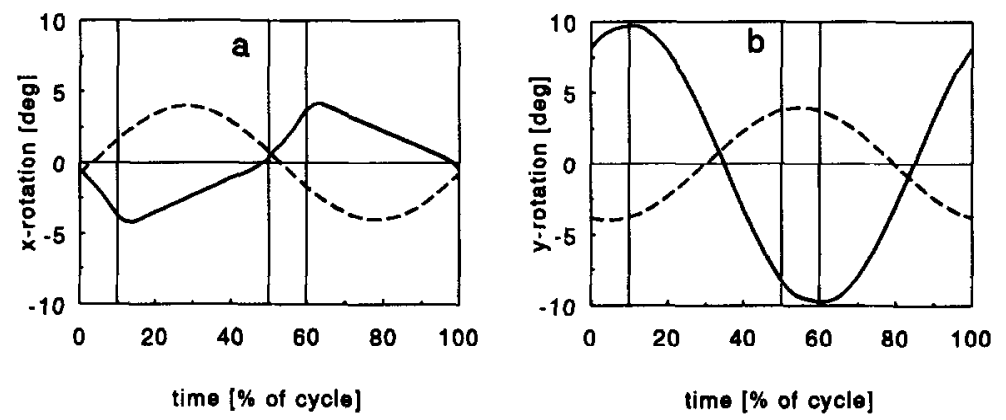

Fig. 6. Rotations around the forward (x)-axis (a) and vertical ( $y$-)axis (b): pelvic rotations constructed with kinematic constraints (solid lines) and predicted HAT rotations (dashed lines).

measured pattern for the $x$-rotation is a zero rotation at or just after heel contact and a maximum rotation of about $5^{\circ}$ just after toe-off.

The predicted HAT rotations are shown in the same figure. The rotation around the forward ( $x$-)axis is more or less contrary to the pelvic $x$-rotation [Fig. 6(a)]. By rotating this way the HAT is moved towards the direction of the supporting leg, thus adding to the mechanisms that keep the body in balance during walking. The rotation of the HAT segment around the vertical ( $y$-)axis is also inverse to the pelvic $y$-rotation [Fig. 6(b)]. This can also be observed in normal walking (e.g. Inman et al., 1981) where the arms are swung contrary to the legs. The effect of this inverse rotation is that the variation in the vertical angular momentum of the total body is minimal.

The estimated ground reaction forces for this movement of the segments model are compared in Fig. 7 with measured data from Winter (1987). The estimated vertial force component shows the characteristic pattern of weight acceptance and push-off. The agreement is reasonably good when the trends are compared, although the amplitude of the measured vertical accelerations is somewhat larger. This can partly be explained with the effect of smoothing functions, which were necessary when determining the segmental accelerations numerically, and partly with inevitable modeling deviations. The forward force component also shows a good agreement, although the assumption that no counteracting force between both feet exists during the double support phase results in an underestimation.

Figure 8 shows the resulting moments of force at the major joints. The hip abduction moment of force reflects the vertical ground reaction force, which is not surprising since the foot is placed under the body during the support phase. The largest hip flexion/ extension moments occur during the double support phase: when both feet are on the floor, the body is in its most stable position to control the rotation of the HAT segment where most of the body mass is located. The knee extension moment controls the knee flexion during double support and with the ankle plantar flexion moment some of the energy, needed for the push-off, is generated.

\section{DISCUSSION}

The estimated rotations resulting from the construction, reconstruction and prediction agree well with expectations based on the mechanics of walking. Moreover, there is a good agreement with measured data, reported in the literature, at least when the tendencies are considered. A sensitivity analysis showed that the fatigue criterion [equation (3)] only had a small effect on the constructed and reconstruc- 


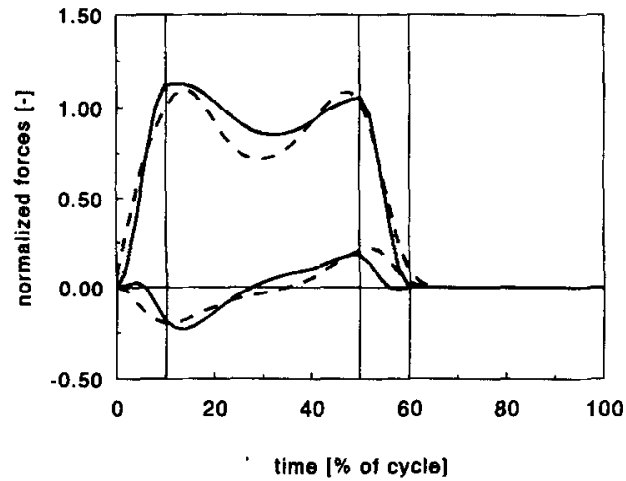

Fig. 7. Estimated forward and vertical ground reaction forces (solid lines), $v=1.4 \mathrm{~m} \mathrm{~s}^{-1}$, normalized with the body weight, compared with measured data from Winter (1987) (dashed lines), $v=1.39 \mathrm{~m} \mathrm{~s}^{-1}$, ensemble average 19 subjects.

ted rotations. However, the agreement between expectations and the predicted rotations (the HAT rotations around the forward and vertical axes) does not prove the validity of the fatigue criterion, it merely shows that this type of criterion yields sensible results for this type of movement.

Relatively small joint angles with an amplitude of about $5^{\circ}$ have a large impact on the dynamic equilibrium of the segments model, as is shown with the reconstruction of the hip adduction and the HAT rotation with the dynamic constraints. The imbalance moment of force can be viewed as an overall measure for the accuracy of the measured data and segments model. A good estimate of the joint moments of force is only guaranteed when this imbalance moment is small; the measuring system should be such that the small angles are still measured accurately. Unfortunately, in most reported gait analysis studies, this dynamic consistency has not been checked.
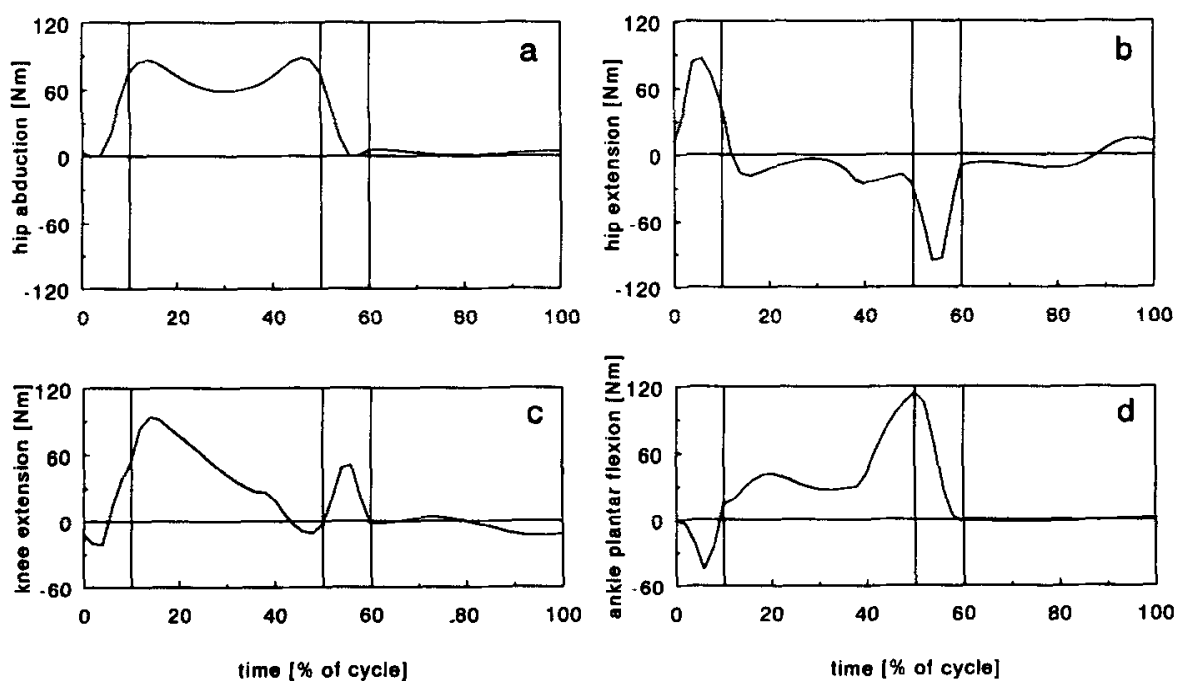

Fig. 8. Calculated hip abduction (a), hip extension (b), knee extension (c) and ankle plantar flexion (d)

moments of force.
mox important balancing mechanism during walking. Due to the large mass and moment of inertia of the trunk (HAT segment), only small rotations and displacements will suffice to reduce the effect of deviations in the walking pattern.

The displacements of the segments relative to the floor and the pelvic rotations are, due to the kinematic constraints, partly determined by the foot shape. A realistic foot shape will improve the results, as numerical experiments with different foot shapes (e.g. a stilt foot with a single contact point and an ellipsoid foot shape) have shown. Likewise, the foot shape that is used affects the estimated ground reaction forces.

The proposed methods may find various applications in gait analysis systems: firstly, it is possible to perform a full three-dimensional analysis, even when no force-plate data is present and only a limited number of joint rotations are measured. Although it will be difficult to assess the quantitative accuracy of the estimated forces and torques, it may well be used for a qualitative comparison. Secondly, in a setting with a three-dimensional measuring system, the constraints provide a valuable check for the accuracy of the measuring system and the segments model. Especially the imbalance moments of force and the difference between the estimated and measured ground reaction forces could be minimized to improve the accuracy.

Finally, the model can be used to predict walking movements in situations where measurements are not feasible, for example, in the design process of new prosthetic and orthotic components or in studying the effect of a variation of parameters. In this case the parameters describing the measured movement may be optimized as well, while the properties of the new design are incorporated in the segments model. Again, it will be difficult to assess the quantitative accuracy of

The results indicate that moving the trunk is an 
the predictions, but the results will yield a qualitative impression of the changes that will take place.

The choice for the inverse method instead of the direct method in the predictive model is somewhat arbitrary. However, the inverse method offers the opportunity of an easy implementation of the kinematic constraints. With the use of the constraints and with the representation of the movement with finite Fourier series, all solutions of the optimization problem will automatically be valid and cyclical walking movements. In fact, the constraints define what is walking.

The main disadvantage of the inverse dynamics method in comparison to the direct dynamics method is often considered to be the amplification of noise by numerically differentiating twice. To avoid smoothing, sometimes the direct dynamics method is chosen to analyse a measured movement, which is then combined with optimization techniques (e.g. Chao and Rim, 1973). However, since this is essentially a curvefitting technique and the information contained in the system (the segments model, the measurements and the equations of motion) is not changed, this method should yield exactly the same results as the inverse dynamics method. When this is not so, the curvefitting is not exact, in which case the direct dynamics method is just a difficult way to implicitly smooth the input movement. When the right precautions are taken in an inverse dynamics method, the amplification of noise can be controlled to reasonable limits.

\section{REFERENCES}

Brand, R. A., Crowninshield, R. D., Wittstock, C. E., Pedersen, D. R., Clark, C. R. and van Krieken, F. M. (1982) A model of lower extremity muscular anatomy. J. biomech. Engng 104, 304-310.

Chandler, R. F., Clauser, C. E., McConville, J. T., Reynolds, H. M. and Young, J. W. (1975) Investigation of the inertial properties of the human body. Report DOT HS-801430. National Technical Information Service, Springfield Virginia.
Chao, E. Y. and Rim, K. (1973) Application of optimization principles in determining the applied moments in human leg joints during gait. J. Biomechanics 6, 497-510.

Chow C. K. and Jacobson, D. H. (1971) An optimal programming study of human gait. Math. Biosci. 10, 103-110.

Crowninshield, R. D. and Brand, R. A. (1981) A physiologically based criterion of muscle force prediction in locomotion. J. Biomechanics 14, 793-801.

Dul, J., Townsend, M. A., Shiavi, R. and Johnson, G. E. (1984a, b) Muscular synergism I-II. J. Biomechanics 17, 663-673, 675-684.

Hardt, D. E. and Mann, R. W. (1980) A five body-three dimensional dynamic analysis of walking. $J$. Biomechanics $15,741-745$.

Hatze, H. (1981a) Quantitative analysis, synthesis and optimization of human motion. Hum. Mvmt Sci. 3, 5-25.

Hatze, H. (1981b) A comprehensive model for human motion simulation and its application to the take-off phase of the long jump. J. Biomechanics 14, 135-142.

Inman, V. T., Ralston, H. J. and Todd, F. N. (1981) Human Walking. William \& Wilkíns, Baltimore/London.

Kadaba, M. P., Ramakrishnan, H. K. ad Wootten, M. E. (1990) Measurement of lower extremity kinematics during level walking. $J$. orthop. Res. 8, 383-392.

Koopman, H. F. J. M. (1989) The three-dimensional analysis and prediction of human walking. Ph.D. thesis, University of Twente, Enschede, The Netherlands.

McMahon, T. A. (1984) Muscles, Reflexes, and Locomotion. Princeton University Press, Princeton, NJ.

Numerical Algorithms Group (1984) NAG Fortran Library Manual, Mark 11, Vol. 3. E04-Minimizing and maximizing a function. Oxford/Downers Grove.

Olney, S. J. and Winter, D. A. (1985) Predictions of knee and ankle moments of force in walking from EMG and kinematic data. J. Biomechanics 18, 9-20.

Pandy, M. G. and Berme, N. (1988) A numerical method for simulating the dynamics of human walking. $J$. Biomechanics 21(12), 1043-1051.

Thunnissen, J. (1993) Muscle force prediction during human gait. Ph.D, thesis, University of Twente, Enschede, The Netherlands.

Vaughan, C. L., Hay, J. G. and Andrews, J. G. (1982a, b) Closed loop problems in biomechanics I-II. J. Biomechanics 15, 197-200, 201-210.

Vink, P. and Karssemeijer, N. (1988) Low back muscle activity and pelvic rotation during walking. Anat. Embryol. 178, 455-460.

Winter, D. A. (1987) The Biomechanics and Motor Control and Human Gait. University of Waterloo Press, Waterloo, Ontario, Canada. 